\title{
Using the correlation property of subharmonic response as an index of cavitation of
}

\author{
microbubbles \\ Men-Tzung Lo ${ }^{1,2}$, Jenho Tsao ${ }^{1}$, and Shinn $\operatorname{Lin}^{2}$. \\ ${ }^{1}$ Graduate Institute of Communication Engineering, National Taiwan University, Taipei, Taiwan. \\ ${ }^{2}$ Medical Group, MICRO-STAR INT'L CO., LTD
}

\begin{abstract}
- in this study, we try to specify whether the weak correlation value of two consecutive Doppler signals for the subharmonics is only related to the reduced observation time, since the subharmonics are generated from the smaller bubbles, the radiation fore effect also reduce the correlation value. Our numerical result presents that most bubbles producing the subharmonics undergo cavitation while the applied pressure is increased to make the subharmonics be significant. In addition, the numerical result shows that there are non-negligible amount of bubbles, which produce the subharmonics and undergo cavitation simultaneously, yielding the spiky Doppler response. Such behavior is demonstrated in experimental Doppler power spectrum for the subharmonics. The inspection of the Doppler power spectrum shows that it doesn't look like the broadened spectrum due to the radiation force. In addition, the experiment with diminishing the radiation force effect showed that the correlation is still very weak. Finally, the rapidly decreased subharmonic intensity under continuous insonification can give us clear evidence that most of bubbles producing the subharmonics would be destroyed while they are moving through the sample volume.
\end{abstract}

\section{INTRODUCTION}

Our previous research [1] demonstrated that when the subharmonics oscillation is significant, the correlation between the two consecutive samples of the subharmonics is excessively weak. We accused the weak correlation of reduced observation time. The previous numerical study demonstrated that under the insonified frequency $\mathrm{f}_{\mathrm{e}}$, except for the bubble with the resonance frequencies of $2 f_{e}$ and $f_{e}$, the subharmonic onset threshold increases with the bubble size. Therefore, when the bubble size is distributed, unlike the

This work is supported partly by the National Science Council, Taiwan, ROC. (NSC93-2213-E002-082) fundamental, which are dominated by larger bubbles, the smaller bubbles have dominant influence on the subharmonic components. The radiation force [2] should have un-negligible effect on the reduced correlation of two consecutive Doppler signals represented as $\overline{R_{x}\left(T_{s}\right)}\left(T_{s}\right.$ : pulse repeat time), since it accelerates the smaller bubbles more than larger bubbles. In this study we try to specify whether the excessively weak $\overline{R_{r}\left(T_{s}\right)}$ is induced chiefly by the reduced observation time rather than radiation force effect, and the reduced observation time is related to cavitation effect.

In this study, based on the numerical onset and cavitation thresholds, we demonstrated that if the acoustic pressure is increased to exceed $250 \mathrm{Kpa}$, over one half of the bubbles that can generate significant subharmonics will undergo cavitation while they are moving through the sample volume. In addition, through the beam gain function, we demonstrated the probability distribution of the observation time to show how short the observation time of the subharmonics would be. In experimental results, the Doppler spectrum of the subharmonics doesn't look like the broadened spectrum due to the radiation force. It contains non-negligible very wideband components due to the spiky Doppler response, which can be expected according to our numerical probability distribution of observation time. In addition, according to our previous mathematical result, we can develop an experiment with the radiation force effect diminished to observe whether $\overline{R_{x}\left(T_{s}\right)}$ is still weak Finally, the variation of the intensity of the subharmonics versus the insonified time would be presented to show that the bubbles generating the subharmonics do undergo cavitation.

\section{NUMERICAL STUDY}

\section{A. Most bubbles undergo cavitation}

In previous study, we have given the onset and cavitation thresholds for different bubble radius 
under a fixed driving frequency by means of numerical solution to the Rayleigh-Plesset (RPNNP) equation. By inspection, the behavior of onset threshold showed that the number of the categories, classified according to bubble size, which can generate significant subharmonic oscillations, increases with the acoustic pressure. However, according to the simulated cavitation threshold, it seems that most of the bubbles that can generate substantial subharmonics would be destroyed simultaneously under high acoustic pressure. Assuming that the bubble radius is distributed over [1 $\mu \mathrm{m}, 3.4 \mu \mathrm{m}]$ uniformly, for different applied pressure ranging from 200 to 450 Kpa, Fig. 1 demonstrates the ratio of the number of the microbubbles whose cavitation thresholds are below the applied pressure to the total number of bubbles, whose corresponding onset thresholds are below the applied pressure. According to Fig. 1, it can be found that if the acoustic applied pressure exceeds $250 \mathrm{Kpa}$, over one half of the bubbles which can generate significant subharmonics, will undergo cavitation while they are moving through the non-uniformly illuminated sample volume.

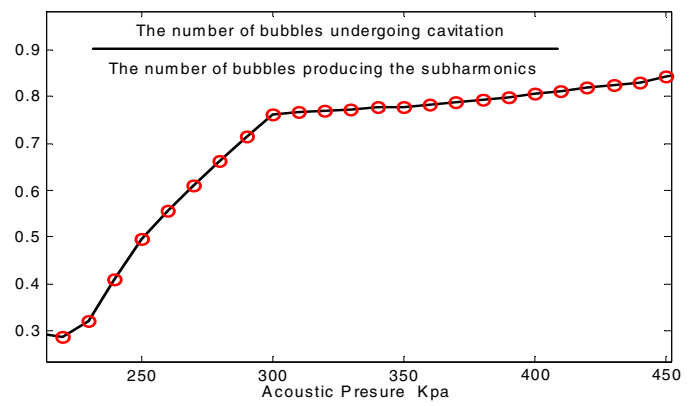

Fig. 1. The ratio of the number of microbubbles undergoing cavitation to the number of bubbles that the applied pressure is above their corresponding onset thresholds.

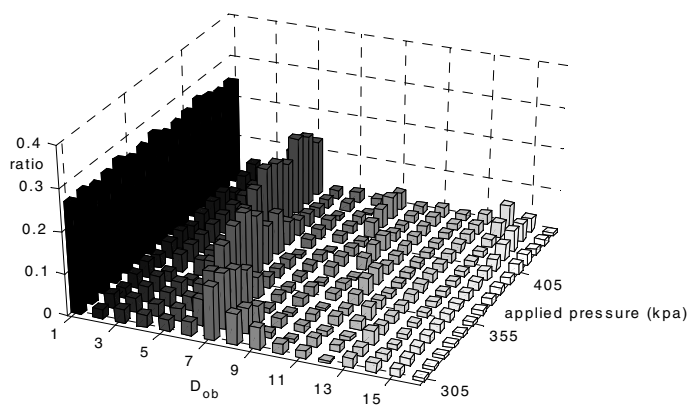

Fig. 2. The probability density functions of $D_{o b}$ when different acoustic pressures are applied.

\section{B. The probability distribution of observation time for the subharmonics under distributed bubble size}

Because the onset threshold for the generation of subharmonics are high, a microbubble cannot be excited to generate the subharmonic signals until it moves to the position where the product of the transmitted beam pattern and the applied pressure is over the corresponding value. While the bubble is moving close to the beam axis of the transducer, the bubble may start to be excited to generate significant subharmonic oscillations, and then it will undergo cavitation and not be detected several firings later, if the cavitation threshold is so high that it approaches the onset threshold. According to Fig. 1, if the applied pressure is high enough to let sufficient bubbles generate the enormous subharmonics, it appears that such bubble, which undergoes cavitation before it reaches the position where the beam pattern is maximum, is the dominant category of the bubbles producing the subharmonics. It follows that the observation time for the subharmonics are reduced by not only onset threshold effect but also cavitation effect.

Assume that concave transducer with radius 10 $\mathrm{mm}$ is used to emit the transmission, the angle between the beam axis and the flow direction is $\pi / 4$, the focal distance is $20 \mathrm{~mm}$ away from the transducer, the emitted frequency of the transmission is $2.5 \mathrm{MHz}$ and the pulse repeat frequency is $10 \mathrm{KHz}$; the transit time of the particle can be calculate to be $42 T_{s}$ when the fluid velocities is $200 \mathrm{~mm} / \mathrm{sec}$ and moving across twenty percent of the beam width. Under the long burst insonification with applied acoustic pressure $P_{A}$, the microbubble cannot generate the subharmonics until it moves into the area where the product of $P_{A}$ and beam pattern is over the corresponding onset threshold. In addition, the certain bubble will not generate the subharmonics anymore once the insonified pressure is over the corresponding cavitation threshold. Therefore, only when the insonified pressure is between onset and cavitation thresholds, the subharmonics can be observed. Using this criterion, we can count the length of the firing sequence, which can excite the bubble to generate the subharmonics consecutively. The result is denoted as $D_{o b}$ and $D_{o b} \cdot T_{s}$ is regarded as observation time of the subharmonics. We assume that the bubble size in the vessel is distributed over $[1 \mu \mathrm{m}, 3.4 \mu \mathrm{m}]$ uniformly. Since bubble size is distributed, and the onset and cavitation thresholds are size dependent, $D_{o b}$ is a random number and it 
is distributed over $[0,42]$ when fluid velocities are $200 \mathrm{~mm} / \mathrm{sec}$. Exclude the case $D_{o b}=0$, we plot the probability density function of $D_{o b}$ in Fig. 2 when the different pressures $P_{A}$ are applied. According to Fig. 2, we find that most subharmonic components are generated with very short observation time. In addition, it can be observed that the expected value of subharmonic observation time decreases with the applied pressure.

\section{EXPERIMENTAL STUDIES}

\section{A. Obscured Doppler spectrum}

To observe the influence of reduced observation time on spectral Doppler, we conduct the following experiment. In this experiment both the transmitting and receiving transducers are V305 (i.e. T2R2). The applied pressure is set at $800 \mathrm{Kpa}$. The microbubbles in this experiment are free gas with the concentration of $0.6 \mathrm{ml} / 100 \mathrm{ml}$. While the transducers were enabled, the transmitted transducer emitted Gaussian envelope bursts with standard deviation of $10 \mu \mathrm{s}$ at a PRF of $5 \mathrm{KHz}$. The power spectrum of 128 Doppler signals multiplied with Hanning window for each harmonic component was evaluated, and then the results for the subharmonics, the fundamental, and the second-harmonics are plotted as gray scale with the dynamic range of $16 \mathrm{~dB}$ in Fig. 3(a), (b), and (c), respectively. The corresponding power spectrum of 128 Doppler samples of the first realization for different selected harmonics was illustrated in the right of Fig. 3. It can be found that the velocity distribution is concentrated in Doppler power spectrum for the fundamental and second-harmonics, whereas the Doppler power spectrum for the subharmonics was confused by wide band components. We demonstrated the averaged Doppler power spectrum over 144 realizations in Fig. 4. As shown in Fig. 4, the flow velocity components get to be concentrated in the averaged Doppler power spectrum for the subharmonics, even though the contrast between the Doppler shift related to flow velocity and wide band components in Doppler power spectrum for the subharmonics is still weak relative to the fundamental and the second-harmonics. Note that the intensity of the subharmonics is greater than that of the second-harmonics in RF domain under such applied acoustic pressure. However, the normalized power density of the wide band components in Doppler spectrum for the subharmonics is excessively higher than that for the second-harmonics. Therefore, it appears that the source of wide band components in Doppler spectrum for the subharmonics is different from that for the second-harmonics.

According to Fig. 2, it appears that the nonnegligible subharmonic components in RF domain are excited from the bubbles, which produce the subharmonics and undergo cavitation simultaneously. This results in the spiky Doppler response and contributes to the wideband components in Doppler spectrum as shown in Fig. 1
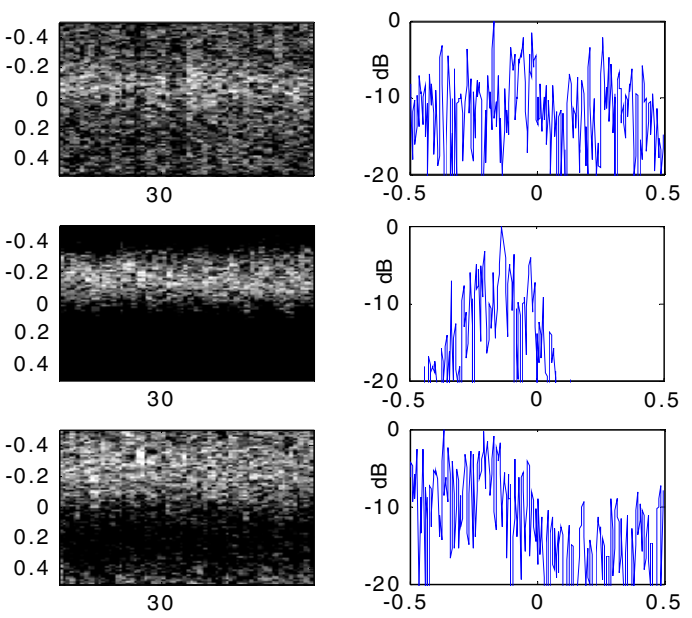

Fig. 3. (left) The spectral Doppler (72 realizations) for the ubharmonics (a), the fundamental (b), and the second harmonics (c), respectively. (right) The corresponding Doppler ower spectrum of first realization.

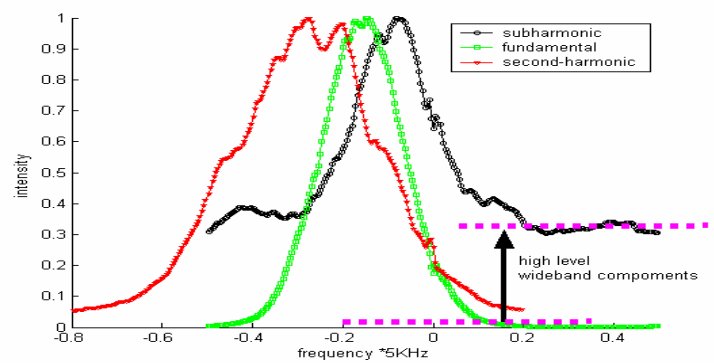

Fig. 4. The averaged Doppler power spectrum for different harmonics.

\section{. Weak correlation value with diminishing radian force effect}

Our previous analysis[1] demonstrates that the mean flow velocity should be under-estimated due to the reduced observation time, since the slower elocity components are weighted more heavily. In $\mathrm{V}$ other words, the estimated mean flow velocity using the subharmonics is smaller than that using the fundamental. On the contrary, relative to the fundamental, the radiation force will increase the estimated mean flow velocity using the 
subharmonics when the bubble is away from the transducer. Therefore, to diminish the radiation force effect, the value of $\overline{R_{x}\left(T_{s}\right)}$ under that the estimated mean velocity using the subharmonics is not larger than that using the fundamental is selected to specify whether the observation time for the subharmonics is excessively reduced. The ratios of estimated phase of $\overline{R_{x}\left(T_{s}\right)}$ for the subharmonics to that for the fundamental under different applied pressure are illustrated in Fig. 5. It can be found that under lower applied pressure such as $0.4 \mathrm{Mpa}$, the ratio of estimated phase of $\overline{R_{x}\left(T_{s}\right)}$ using the subharmonics to that using the fundamental is smaller than 0.5 . Note that the $\overline{R_{x}\left(T_{s}\right)}$ estimated using subharmonics (i.e. 0.28 ) is still much smaller than that using the fundamental (i.e.0.89) when the applied pressure is set to be 0.4 Mpa under which the estimated flow velocity using the subharmonics is smaller than that using the fundamental. Note that the subharmonics has substantial SNR (i.e.over $20 \mathrm{~dB}$ ) under the pressure $0.4 \mathrm{Mpa}$. Therefore, it can be concluded that the excessively weak $\overline{R_{x}\left(T_{s}\right)}$ is induced chiefly by the reduced observation time.

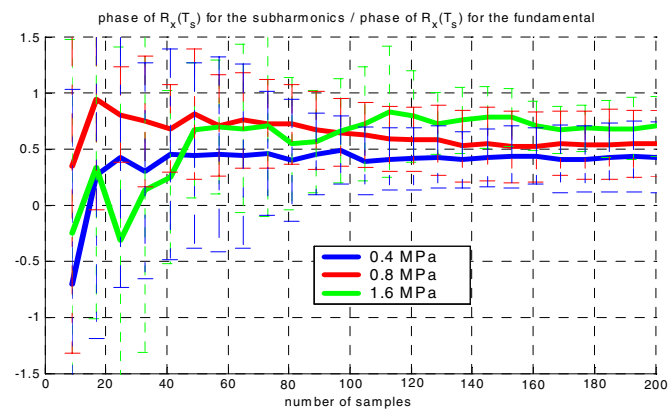

Fig. 5. The mean and standard deviation of 12 estimated ratio of phase of $\overline{R_{x}\left(T_{s}\right)}$ using the subharmonics to that using the fundamental as a function of the number of samples.

\section{Long time response of subharmonics}

Now, we can recognize that the observation time for the subharmonics is excessively short. However, it is still a question whether the cause of the reduced observation time is related to cavitation effect, since without the cavitation effect, only high onset threshold can still reduce the observation time for the subharmonics by itself. To specify the cavitation effect on the Doppler signals of subharmonic furthermore, we performed the following experiment. In this experiment, the microbubbles in the tube is the suspension of Levovist $^{\oplus}$, which has a concentration of about $0.0125 \mathrm{~g} / \mathrm{mL}$. The transmitting transducer emits the Gaussian envelope bursts with standard deviation of $10 \mu \mathrm{s}$ and center frequency of $2.1 \mathrm{MHz}$ at a $\mathrm{PRF}$ of $5 \mathrm{KHz}$ continuously, whereas the receiving transducer was enabled to receive echoes from microbubbles every 12 seconds. Note that both the transmitting and receiving transducers are V305 (i.e. T2R2). We plotted the intensity of selected harmonic component as a function of insonified time in Fig. 6. The mean value and standard deviation of each component in the plot were evaluated from 3 measurements. The applied acoustic pressure in this experiment was set at 1 $\mathrm{MPa}$. It can be observed that the subharmonic component of echoes decreased rapidly with insonified time. However, there wasn't obvious decrement for other harmonics. Therefore, it appears that the dominant fundamental and subharmonic components should be produced by different categories of microbubbles in our experiment. In addition, most of the microbubbles, which can produce subharmonic components, would undergo cavitation easily while they are in the insonified region.

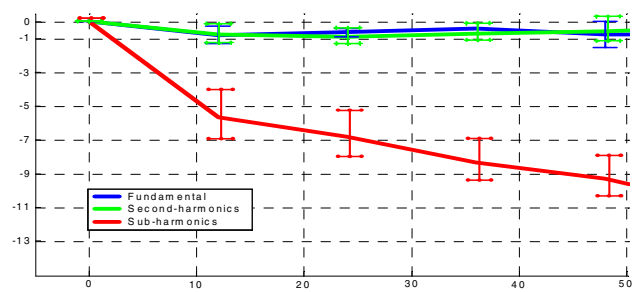

Fig. 6. The backscattering intensity of different harmonics as a function of continuously insonified time.

\section{CONCLUSION}

According to the experimental results, we can exclude the radiation force effect from the cause of excessively weak correlation of two consecutive Doppler signals for the subharmonics. Therefore, the weak correlation should be accused of the reduced observation time chiefly. Consequently, the occurrence of the subharmonics can be used as an indication of undergoing cavitation.

\section{Reference}

[1] Mentzung Lo, Dung-Han Tsai, and Jenho Tsao, "Flow Estimation Using Subharmonics of Microbubbles," Proc. IEEE Ultrason. Symp. pp. 1187-1190, 2003.

[2] P. Tortoli, M. Pratesi, and V. Michelassi, "Doppler Spectra from Contrast Agents Crossing an Ultrasound Field," IEEE Trans, Ultrason., Ferroelect., Freq. Contr., Vol. 47, No. 3, pp. 716-725, 2000.

E-mail:d88942007@ntu.edu.tw 